\title{
FREQUENCY-DEPENDENT DAMPING IN PROPAGATING SLOW MAGNETO-ACOUSTIC WAVES
}

\author{
S. Krishna Prasad ${ }^{1}$, D. Banerjee ${ }^{1}$, and T. Van Doorsselaere ${ }^{2}$ \\ ${ }^{1}$ Indian Institute of Astrophysics, II Block, Koramangala, Bangalore 560 034, India; krishna@iiap.res.in \\ ${ }^{2}$ Center for mathematical Plasma Astrophysics, Mathematics Department, KU Leuven, Celestijnenlaan 200B bus 2400, B-3001 Leuven, Belgium \\ Received 2014 January 13; accepted 2014 May 22; published 2014 June 20
}

\begin{abstract}
Propagating slow magneto-acoustic waves are often observed in polar plumes and active region fan loops. The observed periodicities of these waves range from a few minutes to a few tens of minutes and their amplitudes were found to decay rapidly as they travel along the supporting structure. Previously, thermal conduction, compressive viscosity, radiation, density stratification, and area divergence were identified to be some of the causes for change in the slow wave amplitude. Our recent studies indicate that the observed damping in these waves is frequency-dependent. We used imaging data from the Solar Dynamics Observatory/Atmospheric Imaging Assembly to study this dependence in detail and for the first time via observations we attempted to deduce a quantitative relation between the damping length and frequency of these oscillations. We developed a new analysis method to obtain this relation. The observed frequency dependence does not seem to agree with the current linear wave theory and it was found that the waves observed in the polar regions show a different dependence from those observed in the on-disk loop structures despite the similarity in their properties.
\end{abstract}

Key words: methods: data analysis - methods: observational - Sun: corona - Sun: oscillations

Online-only material: color figures

\section{INTRODUCTION}

Polar plume/interplume regions and extended fan loop structures in active regions are often found to host outward propagating slow magneto-acoustic waves. Besides their contribution to coronal heating and solar wind acceleration, they are important for their seismological applications (King et al. 2003; Marsh et al. 2009; Wang et al. 2009; Van Doorsselaere et al. 2011). The observed periods of the slow waves are of the order of a few minutes to a few tens of minutes. These waves cause periodic disturbances in intensity and Doppler shift and are mostly identified from the alternate slanted ridges in the time-distance maps in intensity (Deforest \& Gurman 1998; De Moortel et al. 2000). However, spectroscopic studies by some authors indicate periodic asymmetries in the line profiles, suggesting the presence of high-speed quasi-periodic upflows, which also produce similar signatures in time-distance maps (De Pontieu \& McIntosh 2010; Tian et al. 2011a, 2011b). This led to an ambiguity in the interpretation of observed propagating features as slow waves. However, later studies found that flow-like signatures are dominantly observed close to the foot points (Nishizuka \& Hara 2011; Tian et al. 2012) and no obvious blueward asymmetries were observed in the line profiles higher in the loops (Krishna Prasad et al. 2012a; Gupta et al. 2012). Results from the recent three-dimensional magneto-hydrodynamic (MHD) simulations by Ofman et al. (2012) and Wang et al. (2013), who report the excitation of slow waves by impulsively generated periodic upflows at the base of the coronal loop, were in agreement with this. Also, the propagation speeds were found to be temperaturedependent for both sunspot (Kiddie et al. 2012) and non-sunspot related structures (Uritsky et al. 2013), in agreement with the slow mode behavior. So, the propagating disturbances observed in the extended loop structures and polar regions can be interpreted as being due to slow waves.

One of the important observational characteristics of these waves is that they tend to disappear after traveling some distance along the supporting (guiding loop) structure.
Their amplitude rapidly decays as they propagate. Thermal conduction, compressive viscosity, optically thin radiation, area divergence, and gravitational stratification were identified as being some of the physical mechanisms that can alter the slow wave amplitude. The gravitational stratification leads to an increase in the wave amplitude, whereas the other mechanisms cause a decrease (see the review by De Moortel 2009 and references therein). Using forward modeling to match the observed damping, it was found that for a slow mode with shorter (five minutes) periodicity, thermal conduction is the dominant damping mechanism and when combined with area divergence it can account for the observed damping even when the density stratification is present (De Moortel \& Hood 2004). They also found that the contribution of the compressive viscosity and radiative dissipation to this damping was minimal. Another study on oscillations with longer periods $(\approx 12$ minutes) traveling along sub-million-degree cool loops suggested that area divergence has the dominant effect over thermal conduction (Marsh et al. 2011). Recently, Krishna Prasad et al. (2012b) had shown that this damping is dependent on frequency. These authors constructed power maps in three different period ranges from which they conclude that longer period waves travel larger distances along the supporting structure while the shorter period waves are damped more heavily. Such frequency-dependent damping was earlier reported by Wang et al. (2002) and Ofman \& Wang (2002) for standing slow waves observed in hot coronal loops. In the present work, we aim to study the quantitative dependence of the damping length of the wave on its frequency. Details on the observations are presented in the next section followed by the analysis method employed and the results obtained. Related theory and the physical implications of the results obtained are discussed in the subsequent sections.

\section{OBSERVATIONS}

Data used in this study are comprised of images taken by the Atmospheric Imaging Assembly (AIA; Lemen et al. 2012) 

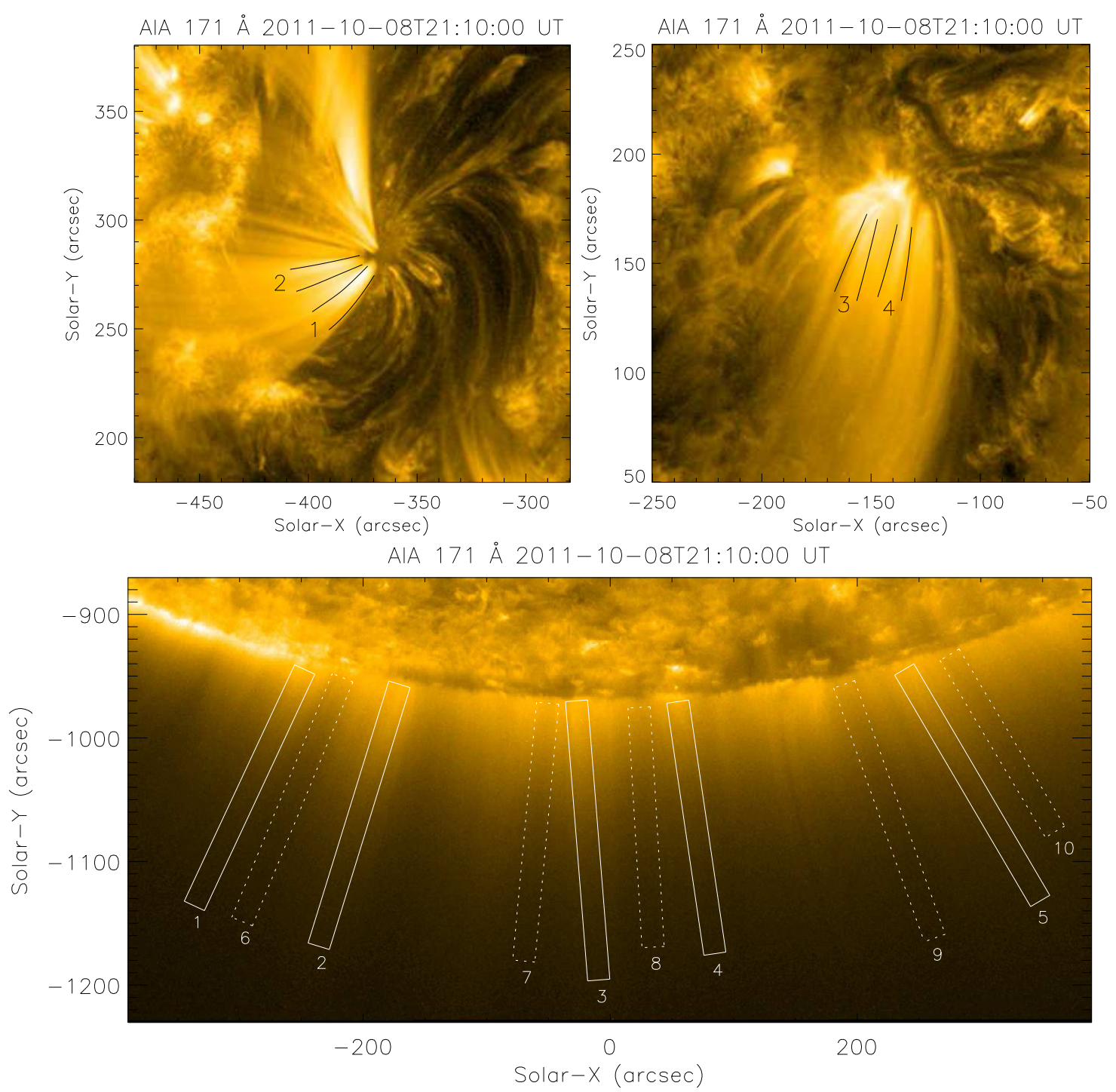

Figure 1. Snapshots of the subfield regions chosen to cover a sunspot (left) and an on-disk plume-like structure (right) and the plume/interplume structures at the south pole (bottom). Locations of the selected structures from these regions are also marked.

(A color version of this figure is available in the online journal.)

on board the Solar Dynamics Observatory (SDO; Pesnell et al. 2012) in two different Extreme Ultra-Violet (EUV) channels centered at $171 \AA$ and $193 \AA$. Full-disk images of $3 \mathrm{hr}$ duration, starting from 21:10 UT on 2011 October 8, were considered. The cadence of the data is $12 \mathrm{~s}$. The initial data at level 1.0 were processed to correct the roll angles and the data from different channels were brought to a common center and common plate scale following the standard procedure using aia_prep.pro routine (ver. 4.13). The final spatial extent of each pixel is $\approx 0^{\prime \prime} 6$.

Subfield regions were chosen to cover loop structures over a sunspot, an on-disk plume-like structure, and the plume/interplume structures at the south pole. The imaging sequence in each of these regions was co-aligned using intensity cross-correlation, taking the first image as the reference. A snapshot for each of the selected on-disk regions and the polar region are shown in Figure 1.

\section{ANALYSIS AND RESULTS}

Four loop structures, two from a sunspot region and another two from an on-disk plume-like structure, were selected to represent the on-disk region and several plume and interplume regions at the south pole, were selected to represent the polar region for this study. The selection of these structures was made on the basis of cleanliness of the propagating oscillations by looking at the time-distance maps. Figure 1 displays the selected loop structures on-disk and the plume/interplume structures at the south pole. The width of the selected loop structures varied from 7 to 19 pixels and that of the plume/interplume structures was fixed at 30 pixels. The enhanced time-distance maps for the loop structure labeled " 1 " in the top panel of Figure 1, are shown in Figure 2, for both the AIA channels. A background constructed from the 300 point ( 60 minutes) running average in time has been subtracted from the original and the resultant is normalized with the same background to produce these enhanced time-distance maps. These maps clearly show alternate slanted ridges of varying intensity due to outward propagating slow waves. Ridges are not visible throughout the length of the chosen loop segment due to rapid decay in the slow wave amplitude as it propagates along the structure. However, they are present for the entire duration of the data set. Another interesting feature visible in these maps is the 


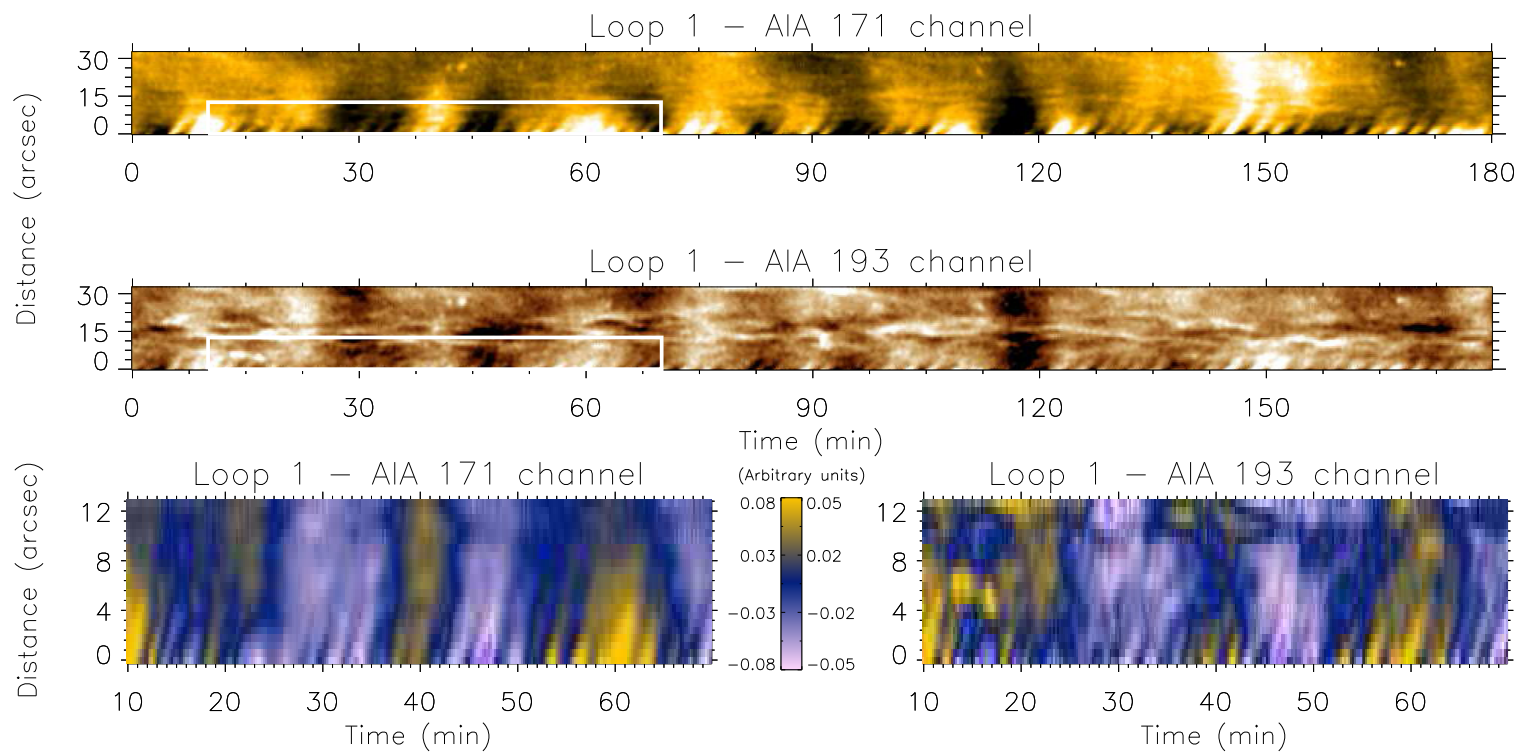

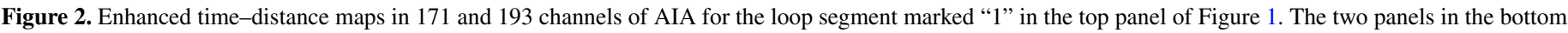
row display the region outlined with a box in the top two panels to present a zoomed-in view.

(A color version of this figure is available in the online journal.)

presence of multiple periodicities. Two different periods, one less frequent (longer period) and another more frequent (shorter period), are apparent from these maps which can be more clearly seen from the zoomed-in view presented in the bottom panels of Figure 2. These shorter and longer periods roughly correspond to a periodicity of 3 and 22 minutes. There can be additional periods present in the signal which may not be not visually evident from these maps.

Our main aim is to measure the damping lengths of these waves at different periods and study the relation between them. Ideally one would look for measuring the damping lengths directly from the decaying wave amplitude along the loop, at a particular instant. However, the damping in these waves is so rapid that we are hardly able to observe more than a cycle. This makes the direct measurements difficult. The simultaneous presence of multiple periods is another obstacle. To overcome these issues, we transformed the original time-distance maps into period-distance maps by replacing the time series at each spatial position with its power spectrum. These maps contain the oscillation power at different periods for each spatial position. In this way, we can not only isolate the power in different periods, but can also trace the spatial decay in amplitude from the corresponding variation in power. Figure 3 displays the period-distance maps generated from the time-distance maps for loop 1. A notable feature in these maps is the presence of more power in longer periods up to larger distances as observed by Krishna Prasad et al. (2012b).

Now to identify all the periods present, we constructed an average light curve from the bottom five pixels of the structure and used it to generate a template power spectrum (see Figure 4). The peak periods and their respective widths were then estimated using a simple routine (gt_peaks . pro) available with the solar software. At each peak identified, we constructed a bin of width determined by the width of the peak and computed the spatial variation of the total power in that bin from period-distance maps. Taking the square root of the power as amplitude of the oscillation, the amplitude decay at a particular period is fitted with a function of the form $A(y)=A_{0} e^{-y / L_{d}}+C$ to compute the damping length $L_{d}$ at that period. The template power spectrum constructed for loop 1 in the 171 channel is shown in the left panel of Figure 4. All the identified peaks and their respective widths are marked with solid and dashed lines in this plot. Note that the routine we used to estimate the widths of peaks gives very rough estimates which can be significantly different from the actual widths. However, this is good enough to isolate the power in individual periods and is far better than the regular way of summing the power in predefined period ranges without having the knowledge of the peak frequencies present in the data. The amplitude decay and the fitted function corresponding to all the identified periods are shown in the right panel of the figure. Different symbols (colors) are used to show the data for different periods. Corresponding plots for 193 channel are shown in the bottom panel. In the plots depicting the amplitude decay, the data for each period are offset by a constant value (50 for 171 and 5 for 193 channels) from the preceding period to avoid cluttering. The computed damping lengths from each period are listed in the plot legend along with the respective errors obtained from the fit. The exact fit parameters $\left(A_{0}, L_{d}\right.$, and $C$ ) estimated for all the periods obtained from the data are listed in Tables 2 and 3 of the Appendix. The exponential fits are quite good for the amplitude decay in most of the cases, but occasionally we find some random variations (bumps) in the amplitude leading to very high damping lengths. We found that this is caused by the contamination from background structures. To eliminate such data, we considered the damping lengths larger than the length of the supporting structure as unreliable. Thus, we measured the damping lengths at different periods. Only those periods between 2 minutes and 30 minutes were considered, keeping the total duration $(3 \mathrm{hr})$ and cadence $(12 \mathrm{~s})$ of the data set in mind.

We combined the results from all the four loop structures on-disk and plotted the measured damping lengths against the period. This allows us to evenly populate the frequency spectrum since the loops with different physical conditions support different frequencies. A similar procedure had been followed for the plume/interplume regions at the south pole except that the time-distance maps in the polar region are constructed by making artificial slits of 30 pixels (fixed) width to avoid the 

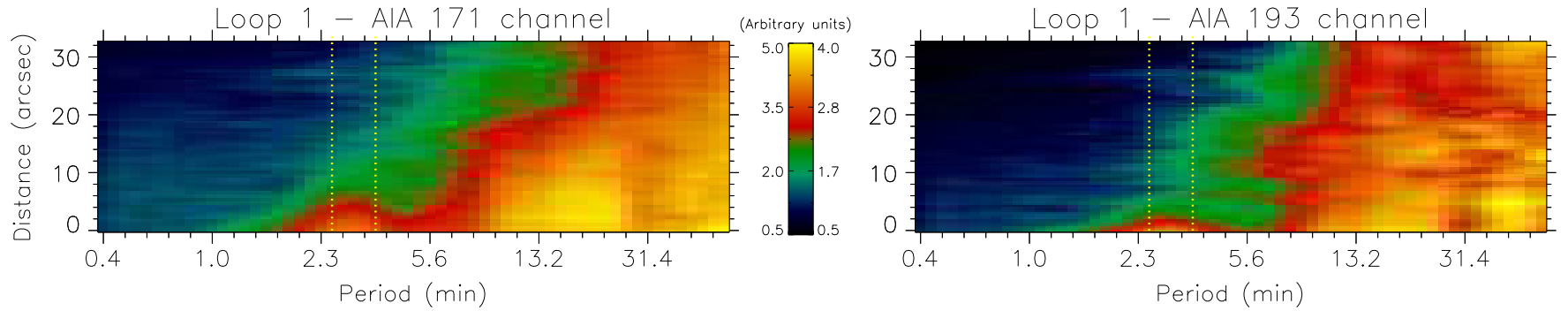

Figure 3. Period-distance maps in $171 \AA$ And $193 \AA$ channels of AIA generated from the time-distance maps for loop 1 . Dotted lines enclose the power at the 3 minute period.

(A color version of this figure is available in the online journal.)
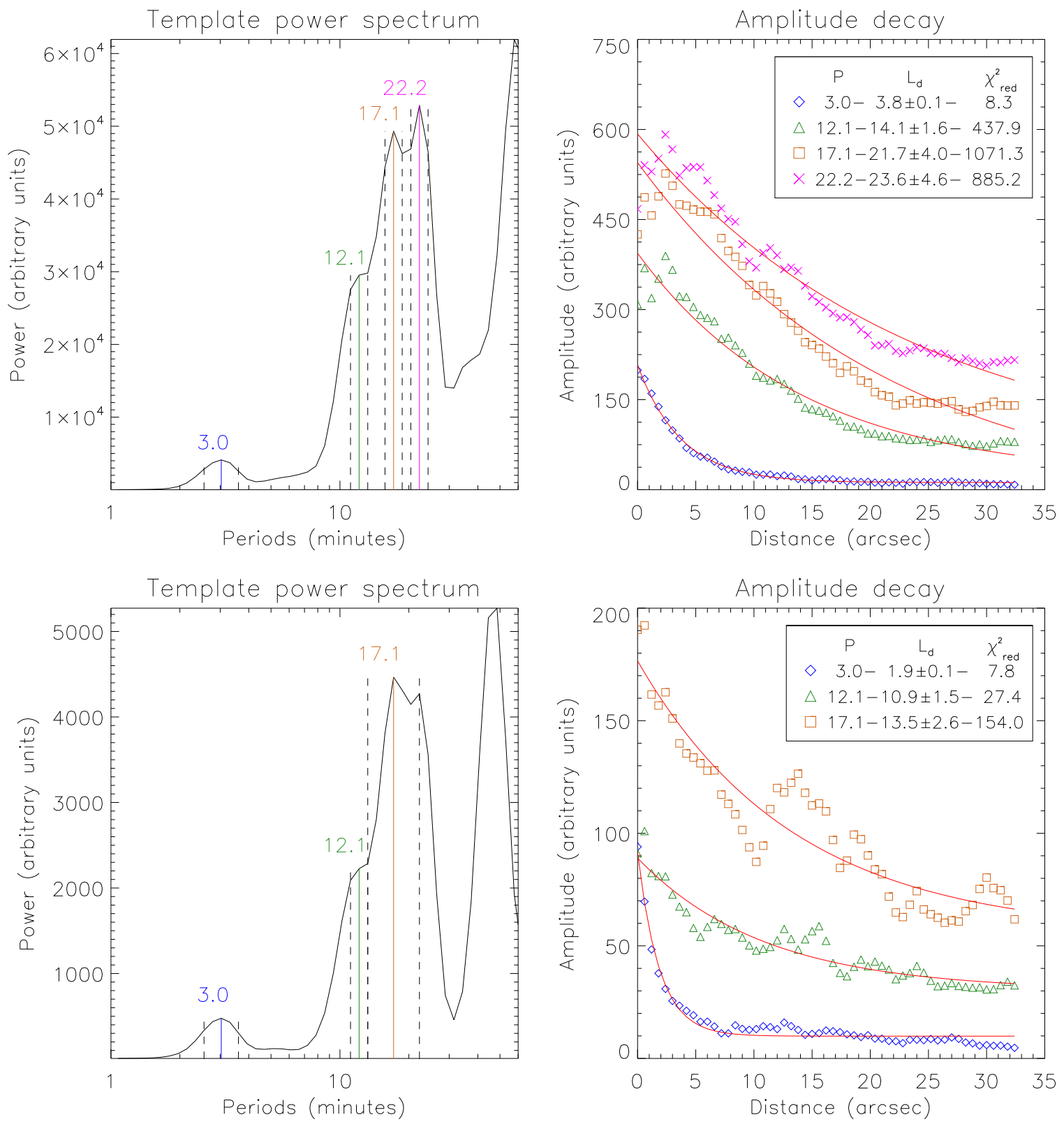

Figure 4. Left: template power spectra constructed for loop 1. The solid vertical lines mark the identified peaks and the dashed lines mark their respective widths. Periodicities of individual peaks are listed above them in minutes. Right: amplitude decay and the fitted model (solid line) for all the identified periods. Different symbols/colors were used to distinguish the different periods. A constant offset was added to the amplitudes to avoid cluttering between different periods. Damping lengths (in arcseconds) obtained from the fit and the corresponding reduced $\chi^{2}$ values are listed for each period in the plot legend. Top and bottom panels correspond to the data from 171 and 193 channels, respectively.

(A color version of this figure is available in the online journal.) 

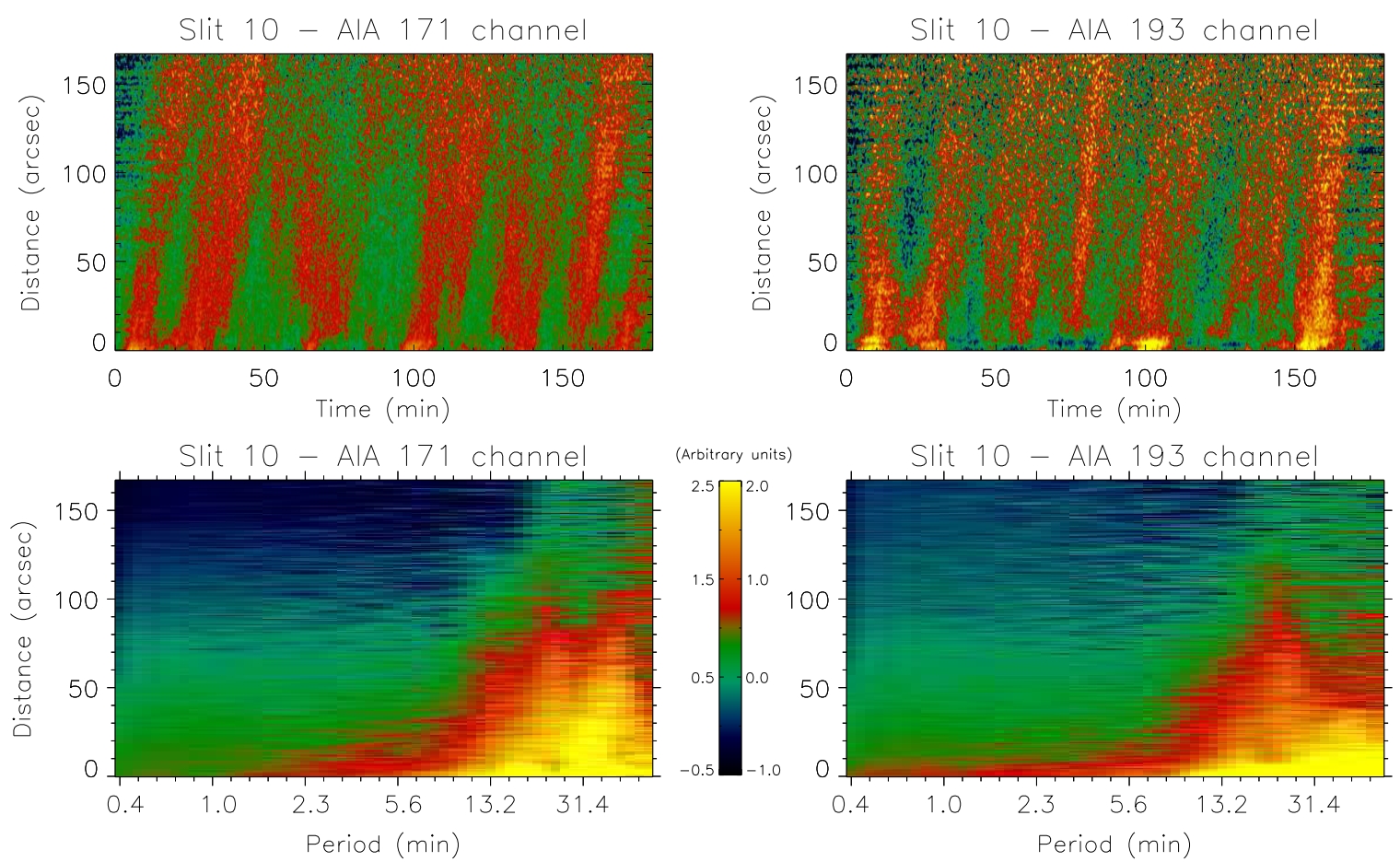

Figure 5. Enhanced time-distance maps (top) and period-distance maps (bottom) in 171 and 193 channels of AIA, generated from the interplume region marked by slit 10 in Figure 1.

(A color version of this figure is available in the online journal.)

effect of jets (Krishna Prasad et al. 2011). The time-distance maps and the corresponding period-distance maps constructed from the interplume region denoted by slit 10 (see Figure 1) are shown in Figure 5 for both the channels. The propagating intensity disturbances are clearly seen in these images, but for some of the slit locations in 193 (slits 1, 2, 6, 7, and 8 in Figure 1) the signal is very poor and we do not see any clear signature of these disturbances. This is possible because the 193 channel looks at relatively hotter plasma (1.25 MK) compared to the typical temperatures of plume/interplume regions ( $<1 \mathrm{MK})$. So the data from these locations are discarded in our final analysis. None of the data are discarded from the 171 channel. Figure 6 displays the plots for damping length versus period in log-log scale. The top two panels correspond to the results from ondisk structures and the bottom two from polar regions. Different symbols (colors) are used to separate the data from sunspot and plume-like structure and plume interplume regions. Damping lengths are measured in arcseconds and periods are measured in seconds. In all the panels, the overplotted solid lines represent a linear fit to the data. The slope of the line and the uncertainty in estimating it are written in the respective panels. There are fewer data points for the on-disk region because of the limited data. Clearly, the on-disk and polar regions show a different dependence of damping length on frequency.

\section{THEORY}

In this section, we study the theoretical dependence of the damping length on the frequency of the slow wave by considering different damping mechanisms separately. To perform this, we follow the one-dimensional linear MHD model of De Moortel \& Hood $(2003,2004)$ and extend it to discuss the frequency dependence. This model is applicable under the assumptions that the magnetic field lines are straight, plasma- $\beta$ is much less than unity, and the amplitude of the oscillations are small. The one-dimensional form of the basic MHD equations for the slow waves can be written as

$$
\begin{gathered}
\frac{\partial \rho}{\partial t}=-\frac{\partial}{\partial z}(\rho v) \\
\rho \frac{\partial v}{\partial t}+\rho v \frac{\partial v}{\partial z}=-\frac{\partial p}{\partial z}-\rho g+\frac{4}{3} \eta_{0} \frac{\partial^{2} v}{\partial z^{2}} \\
\frac{\partial p}{\partial t}+v \frac{\partial p}{\partial z}=-\gamma p \frac{\partial v}{\partial z}+(\gamma-1) \frac{\partial}{\partial z}\left(k_{\|} \frac{\partial T}{\partial z}\right) \\
-(\gamma-1)\left[\rho^{2} \chi T^{\alpha}-H_{0}\right] \\
p=\frac{1}{\tilde{\mu}} \rho R T,
\end{gathered}
$$

where $p, \rho, v$, and $T$ are pressure, density, velocity, and temperature, respectively. $R$ is the gas constant and $\tilde{\mu}$ is the mean molecular weight. The second and third terms on the right hand side of Equation (2) represent the gravitational and viscous forces and those of Equation (3) represent the energy losses due to thermal conduction and optically thin radiation. In these terms, $g$ is acceleration due to gravity, $\eta_{0}$ is coefficient of compressive viscosity, $k_{\|}$is thermal conductivity parallel to the magnetic field, $\chi$ and $\alpha$ are constants under the approximation of a piecewise continuous function for optically thin radiation, and $H_{0}$ is the coronal heating function. In the following subsections, we use appropriate forms of these equations to study the effect of individual damping mechanisms on slow waves and investigate the frequency dependence of damping length. 

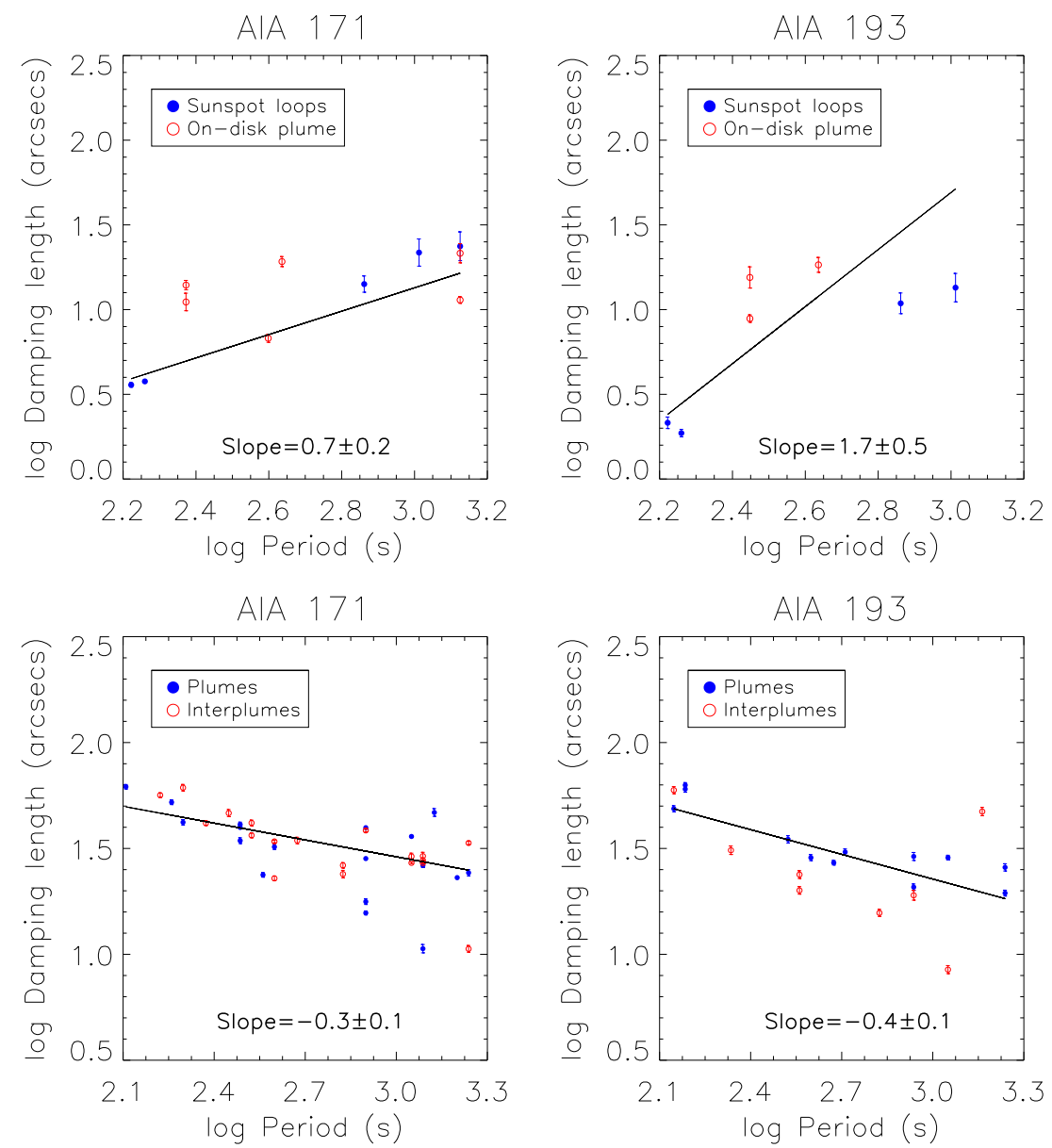

Figure 6. Frequency dependence of damping length for slow waves observed in loop structures on-disk (top) and in polar plume/interplume regions (bottom). Overplotted straight lines represent the linear fit. The slope of the line and the uncertainty in its estimate are written in the respective panels. Different symbols/colors correspond to different data as denoted in the respective legends.

(A color version of this figure is available in the online journal.)

\subsection{Thermal Conduction}

As the slow wave propagates, energy is lost due to thermal conduction which results in a decay of its amplitude. By considering the thermal conduction as the only damping mechanism, we linearized the basic MHD equations and assumed the perturbations in the form $\exp [i(\omega t-k z)]$, to obtain the following dispersion relation for the slow waves

$$
\omega^{3}-i \gamma d k^{2} \omega^{2} c_{s}^{2}-\omega k^{2} c_{s}^{2}+i d k^{4} c_{s}^{4}=0,
$$

where $c_{s}$ is the adiabatic sound speed given by $c_{s}^{2}=\gamma p_{0} / \rho_{0}$ and $d$ is the thermal conduction parameter defined as $d=$ $\left(\left((\gamma-1) k_{\|} T_{0}\right) /\left(\gamma c_{s}^{2} p_{0}\right)\right) . p_{0}, \rho_{0}$, and $T_{0}$ are the equilibrium values of pressure, density, and temperature. The damping length of a propagating velocity perturbation of the form $\exp [i(\omega t-k z)]$ is given by the reciprocal of the imaginary part of $k$. So, to solve for $k$, we simplify the dispersion relation by approximating the thermal conduction at its lower and upper limits. In the lower thermal conduction limit $(d \omega \ll 1)$, Equation (5) reduces to

$$
k=\frac{\omega}{c_{s}}-i \frac{d \omega^{2}}{2 c_{s}}(\gamma-1)
$$

which gives the damping length $L_{d} \propto 1 / \omega^{2}$. This implies that the damping length of slow waves increases with the square of the wave period in the lower thermal conduction limit. Similarly, if we consider the higher thermal conduction limit $(d \omega \gg 1)$, the solution becomes

$$
k=\gamma^{1 / 2} \frac{\omega}{c_{s}}-i \frac{\gamma-1}{2 d \gamma^{3 / 2} c_{s}},
$$

which gives the imaginary part of $k$ independent of $\omega$. Thus, in the limit of higher thermal conduction, the damping in slow waves is frequency-independent.

\subsection{Compressive Viscosity}

The viscous forces lead to the dissipation of energy and therefore reduce the slow wave amplitude. To understand the effect of compressive viscosity quantitatively, we solved the relevant linearized MHD equations assuming all the perturbations are in the form $\exp [i(\omega t-k z)]$, which resulted in the following expression for wave number $k$

$$
k=\frac{\omega}{c_{s}}-i \frac{2}{3} \frac{\eta_{0} \omega^{2}}{\rho_{0} c_{s}^{3}} .
$$

The imaginary part of this expression indicates that the amplitude of the slow wave decreases due to compressive viscosity and the decay lengths are proportional to the square of the wave period. A similar dependence was earlier reported by Ofman et al. (2000). 


\subsection{Optically Thin Radiation}

Energy dissipation due to radiation also causes a decay in wave amplitude. By retaining the radiation term and removing other dissipative terms in the basic MHD equations, one can obtain the dispersion relation for slow waves as

$$
k=\frac{\omega}{c_{s}}-i \frac{r_{p}}{c_{s}}
$$

under the linear regime for perturbations of the form $\exp [i(\omega t-$ $k z)$ ]. Here $r_{p}$ is the radiation parameter defined as $r_{p}=$ $\left(\left((\gamma-1) \rho_{0}^{2} \chi T_{0}^{\alpha}\right) /\left(\gamma p_{0}\right)\right)$. The reciprocal of this parameter has the dimension of time and gives the radiation timescale. According to Equation (9), the damping in slow waves due to optically thin radiation is frequency-independent.

\subsection{Gravitational Stratification}

In contrast to the other mechanisms discussed so far, the gravitational force stratifies the atmosphere which leads to an increase in the slow wave amplitude as it propagates outward. Assuming the initial perturbations of the form $\exp [i(\omega t-k z)]$, we solved the linearized MHD equations to obtain

$$
k=i \frac{1}{2 H} \pm \frac{1}{c_{s}} \sqrt{\omega^{2}-\omega_{c}^{2}} .
$$

Here $H$ is the gravitational scale height given by $H=p_{0} / \rho_{0} g$ and $\omega_{c}$ is the cut-off frequency defined as $\omega_{c}=g \gamma / 2 c_{s}$. This relation indicates that for slow waves with frequencies above the cut-off value $\omega_{c}$, the velocity amplitude grows exponentially as $e^{z / 2 H}$ and the growth rate is independent of frequency. The corresponding amplitude of density perturbations, however, varies as $e^{-z / 2 H}$ considering the equilibrium density fall $\propto$ $e^{-z / H}$ due to stratification. Note that this variation still represents a growth in relative amplitude as $e^{z / 2 H}$, similar to that of velocity perturbations and is independent of frequency.

\subsection{Magnetic Field Divergence}

De Moortel \& Hood (2004) studied the effect of the radial divergence and area divergence of the magnetic field on slow waves. The amplitude of slow waves was found to decrease in both cases as they propagate outward. However, it is important to note that it is purely a geometric effect and there is no real dissipation mechanism involved. We solved the linearized MHD equations in the presence of radial divergence and obtained the following expression for the evolution of velocity perturbations

$$
v(r, t)=\sin (\omega t)\left[C_{1} j_{1}\left(\frac{r \omega}{c_{s}}\right)+C_{2} y_{1}\left(\frac{r \omega}{c_{s}}\right)\right],
$$

(a similar expression was obtained by De Moortel \& Hood 2004). Here $r$ is the radial coordinate in the spherical coordinate notation with the Sun at the center, and $j_{1}\left(r \omega / c_{s}\right)$ and $y_{1}\left(r \omega / c_{s}\right)$ are first order spherical Bessel functions. Substituting the spherical Bessel functions with their standard definition, Equation (11) can be written as

$$
\begin{aligned}
v(r, t)= & \sin (\omega t)\left[C_{1}\left(\frac{\sin \left(\frac{r \omega}{c_{s}}\right)-\frac{r \omega}{c_{s}} \cos \left(\frac{r \omega}{c_{s}}\right)}{\left(\frac{r \omega}{c_{s}}\right)^{2}}\right)\right. \\
& \left.-C_{2}\left(\frac{\cos \left(\frac{r \omega}{c_{s}}\right)+\frac{r \omega}{c_{s}} \sin \left(\frac{r \omega}{c_{s}}\right)}{\left(\frac{r \omega}{c_{s}}\right)^{2}}\right)\right] .
\end{aligned}
$$

Table 1

Dependence of Damping Length on Period of Slow Waves

\begin{tabular}{lcc}
\hline \hline Physical Mechanism & $\begin{array}{c}\text { Amplitude Growth of } \\
\text { Density Perturbations }\end{array}$ & $\begin{array}{c}\text { Period Dependence of } \\
\text { Damping Length }\left(L_{d} \propto\right)\end{array}$ \\
\hline Thermal conduction & $e^{-\frac{d \omega^{2}}{2 c_{s}}(\gamma-1) z}$ & $P^{2}$ \\
Lower limit & $e^{-\frac{(\gamma-1) z}{2 d \gamma^{3 / 2} c_{s}}}$ & $P^{0}$ \\
Upper limit & $e^{-\frac{2}{3} \frac{\eta_{0} \omega^{2} z}{\rho_{0} c_{s}^{3}}}$ & $P^{2}$ \\
Compressive viscosity & $e^{-\frac{r p z}{c_{s}}}$ & $P^{0}$ \\
Optically thin radiation & $e^{\frac{-z}{2 H}}$ & $P^{0}$ \\
Gravitational stratification & $R_{\odot} / r$ & $P^{0}$ \\
Magnetic field divergence & & \\
\hline
\end{tabular}

Notes. $P$ is the time period of the oscillation.

a Note the relative amplitude still grows. See Section 4.4 for details.

The constants $C_{1}$ and $C_{2}$ can be determined from the boundary conditions. We chose these constants such that the amplitude of oscillations at the surface $\left(r=R_{\odot}\right)$ is independent of frequency similar to that we assumed for other cases. Substituting $C_{1}=$ $1 / j_{1}\left(R_{\odot} \omega / c_{s}\right)$ and $C_{2}=1 / y_{1}\left(R_{\odot} \omega / c_{s}\right)$, the velocity $v(r, t)$ becomes

$$
\begin{aligned}
v(r, t)= & \sin (\omega t)\left[\frac{R_{\odot}^{2}}{r^{2}}\left(\frac{\sin \left(\frac{r \omega}{c_{s}}\right)-\frac{r \omega}{c_{s}} \cos \left(\frac{r \omega}{c_{s}}\right)}{\sin \left(\frac{R_{\odot} \omega}{c_{s}}\right)-\frac{R_{\odot} \omega}{c_{s}} \cos \left(\frac{R_{\odot} \omega}{c_{s}}\right)}\right)\right. \\
& \left.-\frac{R_{\odot}^{2}}{r^{2}}\left(\frac{\cos \left(\frac{r \omega}{c_{s}}\right)+\frac{r \omega}{c_{s}} \sin \left(\frac{r \omega}{c_{s}}\right)}{\cos \left(\frac{R_{\odot} \omega}{c_{s}}\right)+\frac{R_{\odot} \omega}{c_{s}} \sin \left(\frac{R_{\odot} \omega}{c_{s}}\right)}\right)\right] .
\end{aligned}
$$

It can be shown that the amplitudes of expressions in the numerator varies as $r \omega / c_{s}$ and that in denominator varies as $R_{\odot} \omega / c_{s}$. This gives the overall amplitude variation as $1 / r$ which is frequency-independent. Following the same treatment, area divergence can be shown to behave similarly. Therefore, we can conclude that the damping in slow waves due to magnetic field divergence is frequency-independent.

A summary on the derived frequency dependence of damping length in slow waves is presented in Table 1 for different physical mechanisms. The table also lists the amplitude growth of density perturbations. It may be noted that although the derivations were primarily done for the velocity perturbations, the density (intensity) perturbations due to slow waves are proportional to the velocity perturbations (as can be derived from Equation (1)), and hence the same growth is expected except for the case of gravitational stratification as mentioned in Section 4.4. We did not explore the frequency dependence due to other geometrical effects like loop curvature, offset, and inclination, and other damping mechanisms like phase mixing and resonant absorption, as we believe the damping in slow waves due to these effects is secondary. For instance, De Moortel et al. (2004) studied the damping of slow waves due to phase mixing and mode coupling to the fast wave using a twodimensional model and found that their contributions are not significant enough to explain the observed damping. However, it may be interesting to note that the amplitude decay for some of the periods can be better fitted with a Gaussian decay function $\left(A(y)=A_{0} e^{-y^{2} / L_{d}^{2}}+C\right)$ rather than an exponential function (see Figure 7). A similar behavior was found by Pascoe et al. (2012) in their numerical simulations for propagating kink waves. It was found analytically that the Gaussian damping for kink 

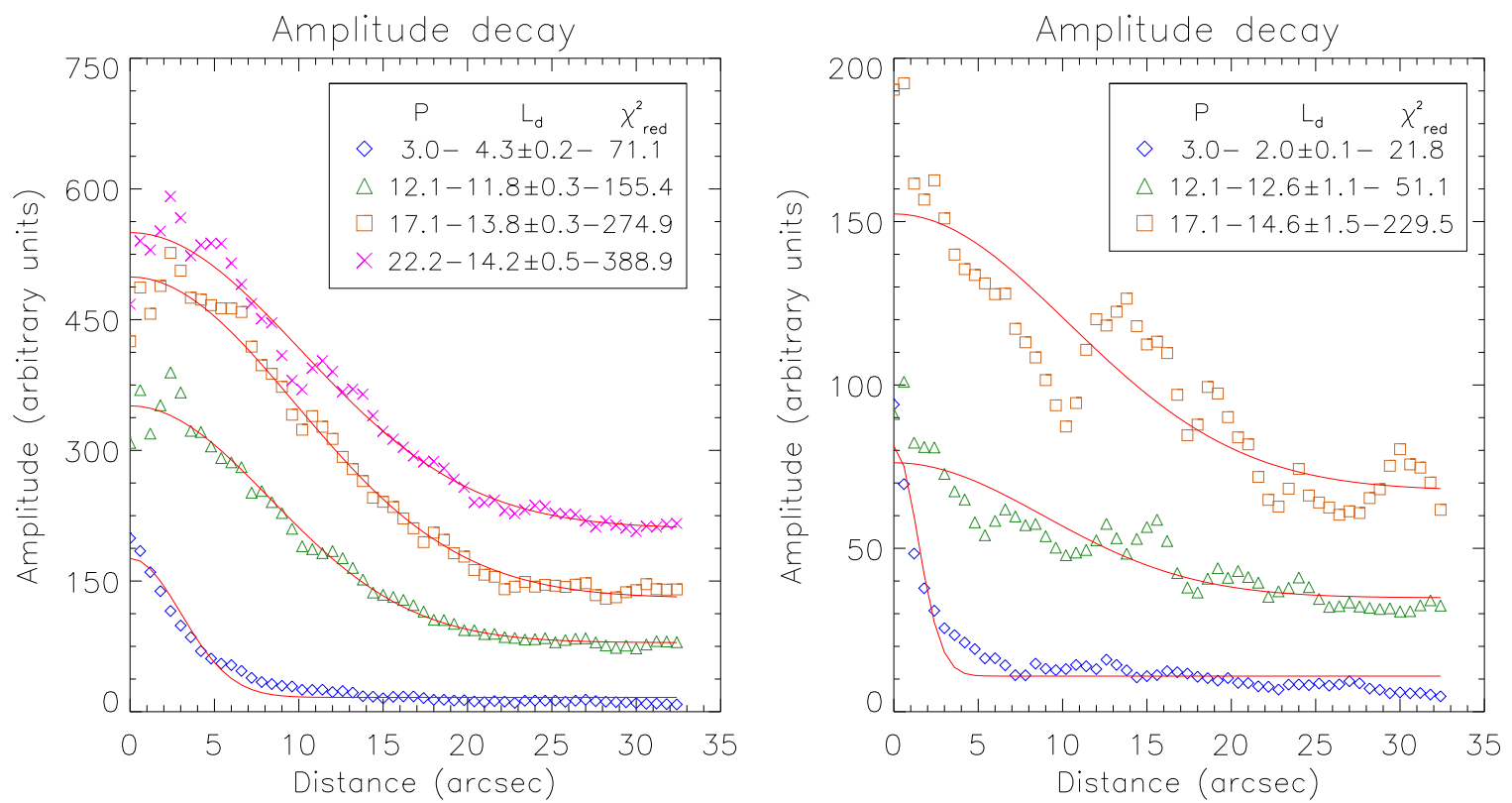

Figure 7. Amplitude decay for the periods shown in Figure 4 fitted with a Gaussian decay model. The damping lengths (in arcseconds) and the corresponding reduced $\chi^{2}$ values are listed in the plot legend. The left and right panels show the results for 171 and 193 channels respectively.

(A color version of this figure is available in the online journal.)

modes is a result of the excitation phase (Hood et al. 2013). In this phase, other modes (besides than the kink mode) are excited and they gradually leak away before the system evolves to the "eigenvalue" state (when it oscillates with the pure kink mode). A consequence of this is that longer wavelengths show the Gaussian damping to greater heights. This also fits with some of our observations where we find that the amplitude decay for the longer periods (and wavelengths) is quite well explained with Gaussian damping. It is unclear, however, if the theory of Hood et al. (2013) for kink modes also holds for slow waves and what physical ingredients are essential for showing this behavior.

\section{DISCUSSION AND CONCLUSIONS}

Damping in slow waves has been studied extensively in polar plumes and active region loops both theoretically and observationally since their first detection. However, studies on the frequency dependence of their damping are limited. Wang et al. (2002) and Ofman \& Wang (2002) studied the frequencydependent damping in standing slow magneto-acoustic waves observed in hot $(T>6 \mathrm{MK})$ coronal loops and found a good agreement between the observed scaling of the dissipation time with the period using their model. They concluded that thermal conduction is the dominant damping mechanism for these waves and the contribution of compressive viscosity is less significant. Theoretical investigations on frequency-dependent damping in propagating slow waves were made by a few authors (Ofman et al. 2000; Nakariakov et al. 2000; Tsiklauri \& Nakariakov 2001). Recently, Krishna Prasad et al. (2012b) report an observational evidence of this using powermaps constructed in three different period ranges. As a follow-up of that work, in this article we studied the quantitative dependence of damping lengths on frequency of the slow waves using period-distance maps.
We selected four loop structures on-disk and about 10 plume/interplume structures in the south polar region that show clear signatures of propagating slow waves. Damping lengths were measured and plotted against the period of the slow wave to find the relation between them. Figure 6 displays the observed dependence of damping lengths on periodicity for the on-disk loop structures and the polar plume/interplume regions in two AIA channels. The slopes estimated from the linear fits are $0.7 \pm 0.2$ (171 channel) and $1.7 \pm 0.5$ (193 channel) for the on-disk regions and are $-0.3 \pm 0.1$ (171 channel) and $-0.4 \pm 0.1$ (193 channel) for the polar regions. The negative slopes obtained for the polar region means the damping lengths for the longer period waves observed in this region are shorter than those for the shorter period waves. Note, however, that in both regions the longer period waves are visible up to relatively larger distances due to the greater availability of power. Considering thermal conduction, magnetic field divergence, and density stratification as the dominant mechanisms that alter the slow wave amplitude, linear theory (see Table 1) predicts the variation of damping length as square of the time period. In a log-log scale, used in Figure 6, this would mean a slope of two. However, as we find here, the slopes estimated from the observations are positive but less than two for the on-disk region and are negative for the polar region. It may be noted that similar negative slopes were found for the polar region, even when the data from plume and interplume regions were plotted separately. This mismatch between the observed values and those expected from the linear theory suggests some missing element in the current theory of damping in slow waves. Perhaps the linear description does not hold well and the slow waves undergo non-linear steepening that causes enhanced viscous dissipation (Ofman et al. 2000). This can be effective for the long-period waves whose amplitudes are relatively larger and possibly can even explain the negative slopes observed in the polar regions. Further studies are required to explore such possibilities and understand the observed 
Table 2

Periodicity, Exponential Fit Parameters, and the Reduced $\chi^{2}$ Values for all the Data from AIA 171 channel

\begin{tabular}{|c|c|c|c|c|c|}
\hline Structure & $\begin{array}{c}\text { Period } \\
\text { (minutes) }\end{array}$ & $\begin{array}{c}A_{0} \\
\text { (Arbitrary Units) }\end{array}$ & $\begin{array}{c}L_{d} \\
(\operatorname{arcsec})\end{array}$ & $\begin{array}{c}C \\
\text { (Arbitrary Units) }\end{array}$ & $\chi_{\text {red }}^{2}$ \\
\hline Loop 1 & 3.0 & $195.32 \pm 1.99$ & $3.77 \pm 0.07$ & $11.95 \pm 0.53$ & 8.32 \\
\hline Loop 1 & 12.1 & $373.84 \pm 13.66$ & $14.14 \pm 1.57$ & $-30.07 \pm 15.95$ & 437.93 \\
\hline Loop 1 & 17.1 & $573.04 \pm 46.96$ & $21.71 \pm 4.01$ & $-128.19 \pm 54.29$ & 1071.35 \\
\hline Loop 1 & 22.2 & $549.66 \pm 51.02$ & $23.65 \pm 4.63$ & $-107.21 \pm 58.02$ & 885.18 \\
\hline Loop 2 & 2.8 & $163.46 \pm 2.70$ & $3.59 \pm 0.11$ & $9.04 \pm 0.69$ & 14.83 \\
\hline Loop 3 & 3.9 & $63.75 \pm 1.30$ & $13.95 \pm 0.89$ & $0.96 \pm 1.36$ & 6.83 \\
\hline Loop 3 & 7.2 & $155.38 \pm 3.76$ & $19.21 \pm 1.31$ & $-9.08 \pm 4.51$ & 25.39 \\
\hline Loop 3 & 22.2 & $275.87 \pm 4.35$ & $11.39 \pm 0.52$ & $19.04 \pm 3.54$ & 85.67 \\
\hline Loop 4 & 3.9 & $13.69 \pm 0.54$ & $11.09 \pm 1.32$ & $7.20 \pm 0.51$ & 1.12 \\
\hline Loop 4 & 6.6 & $32.25 \pm 0.80$ & $6.77 \pm 0.37$ & $10.30 \pm 0.37$ & 2.09 \\
\hline Loop 4 & 22.2 & $140.94 \pm 7.89$ & $21.51 \pm 2.81$ & $-3.37 \pm 9.20$ & 37.76 \\
\hline Slit 1 & 6.1 & $15.70 \pm 0.23$ & $23.72 \pm 0.59$ & $1.50 \pm 0.05$ & 0.55 \\
\hline Slit 1 & 13.2 & $39.83 \pm 0.30$ & $28.33 \pm 0.38$ & $1.60 \pm 0.08$ & 1.11 \\
\hline Slit 1 & 20.4 & $64.69 \pm 0.90$ & $26.30 \pm 0.64$ & $2.42 \pm 0.23$ & 9.23 \\
\hline Slit 2 & 2.1 & $7.59 \pm 0.07$ & $61.83 \pm 1.52$ & $0.86 \pm 0.05$ & 0.11 \\
\hline Slit 2 & 5.1 & $32.99 \pm 0.14$ & $39.73 \pm 0.34$ & $1.49 \pm 0.05$ & 0.34 \\
\hline Slit 2 & 13.2 & $57.21 \pm 0.31$ & $39.62 \pm 0.43$ & $0.92 \pm 0.12$ & 1.61 \\
\hline Slit 2 & 22.2 & $53.16 \pm 0.99$ & $46.75 \pm 1.95$ & $0.44 \pm 0.47$ & 19.26 \\
\hline Slit 3 & 13.2 & $60.78 \pm 0.79$ & $15.68 \pm 0.32$ & $2.55 \pm 0.13$ & 4.45 \\
\hline Slit 3 & 20.4 & $86.50 \pm 2.54$ & $10.63 \pm 0.48$ & $4.23 \pm 0.32$ & 31.98 \\
\hline Slit 3 & 28.8 & $103.80 \pm 2.08$ & $24.27 \pm 0.82$ & $4.89 \pm 0.46$ & 46.10 \\
\hline Slit 4 & 3.3 & $7.24 \pm 0.10$ & $42.02 \pm 1.26$ & $1.17 \pm 0.04$ & 0.17 \\
\hline Slit 4 & 5.1 & $9.55 \pm 0.15$ & $34.41 \pm 1.08$ & $1.28 \pm 0.05$ & 0.35 \\
\hline Slit 4 & 6.6 & $10.44 \pm 0.16$ & $32.16 \pm 0.91$ & $1.31 \pm 0.05$ & 0.33 \\
\hline Slit 4 & 13.2 & $24.97 \pm 0.47$ & $17.73 \pm 0.54$ & $2.02 \pm 0.09$ & 1.76 \\
\hline Slit 4 & 26.4 & $54.65 \pm 0.48$ & $23.02 \pm 0.34$ & $2.02 \pm 0.11$ & 2.28 \\
\hline Slit 5 & 3.0 & $7.02 \pm 0.07$ & $52.24 \pm 1.33$ & $1.20 \pm 0.04$ & 0.11 \\
\hline Slit 5 & 5.1 & $11.39 \pm 0.14$ & $41.03 \pm 1.06$ & $1.81 \pm 0.06$ & 0.35 \\
\hline Slit 5 & 18.7 & $41.99 \pm 0.24$ & $36.01 \pm 0.40$ & $1.51 \pm 0.08$ & 0.88 \\
\hline Slit 6 & 3.9 & $7.43 \pm 0.09$ & $41.63 \pm 1.09$ & $1.10 \pm 0.04$ & 0.15 \\
\hline Slit 6 & 6.6 & $13.09 \pm 0.20$ & $22.84 \pm 0.58$ & $1.48 \pm 0.04$ & 0.39 \\
\hline Slit 6 & 18.7 & $57.04 \pm 0.30$ & $27.03 \pm 0.25$ & $1.94 \pm 0.08$ & 1.04 \\
\hline Slit 6 & 28.8 & $40.15 \pm 0.39$ & $33.55 \pm 0.62$ & $2.50 \pm 0.13$ & 2.17 \\
\hline Slit 7 & 6.6 & $20.37 \pm 0.17$ & $34.12 \pm 0.53$ & $1.28 \pm 0.06$ & 0.40 \\
\hline Slit 7 & 20.4 & $62.27 \pm 1.44$ & $29.08 \pm 1.21$ & $4.00 \pm 0.41$ & 26.00 \\
\hline Slit 8 & 3.3 & $5.58 \pm 0.07$ & $61.22 \pm 2.17$ & $0.90 \pm 0.06$ & 0.09 \\
\hline Slit 8 & 5.6 & $7.75 \pm 0.10$ & $41.73 \pm 1.28$ & $1.20 \pm 0.05$ & 0.19 \\
\hline Slit 8 & 7.9 & $9.90 \pm 0.17$ & $34.48 \pm 1.21$ & $1.38 \pm 0.06$ & 0.44 \\
\hline Slit 8 & 13.2 & $18.98 \pm 0.17$ & $38.47 \pm 0.73$ & $1.12 \pm 0.07$ & 0.46 \\
\hline Slit 8 & 20.4 & $21.98 \pm 0.30$ & $27.80 \pm 0.70$ & $1.86 \pm 0.09$ & 1.10 \\
\hline Slit 8 & 28.8 & $35.96 \pm 0.84$ & $10.61 \pm 0.39$ & $3.60 \pm 0.12$ & 3.51 \\
\hline Slit 9 & 2.8 & $6.51 \pm 0.06$ & $56.43 \pm 1.37$ & $1.37 \pm 0.04$ & 0.08 \\
\hline Slit 9 & 5.6 & $8.24 \pm 0.11$ & $36.46 \pm 0.97$ & $1.30 \pm 0.04$ & 0.20 \\
\hline Slit 9 & 11.1 & $18.76 \pm 0.32$ & $26.31 \pm 0.77$ & $1.66 \pm 0.08$ & 1.17 \\
\hline Slit 9 & 20.4 & $50.68 \pm 0.30$ & $26.99 \pm 0.28$ & $1.63 \pm 0.08$ & 1.07 \\
\hline Slit 10 & 4.7 & $7.03 \pm 0.10$ & $46.45 \pm 1.78$ & $1.33 \pm 0.07$ & 0.17 \\
\hline Slit 10 & 11.1 & $17.83 \pm 0.39$ & $23.94 \pm 0.96$ & $2.44 \pm 0.11$ & 1.60 \\
\hline Slit 10 & 18.7 & $45.65 \pm 0.81$ & $28.99 \pm 1.03$ & $3.92 \pm 0.30$ & 8.27 \\
\hline
\end{tabular}

frequency dependence. Nevertheless, the discrepancy in the results from the on-disk and the polar regions indicates the existence (or dominance) of different damping mechanisms in these two regions possibly due to different physical conditions. It is also possible that the sunspot loops and the on-disk plume-like structures also behave differently, but the current data is limited to make any such conclusions.

We thank the anonymous referee for useful comments. The authors also thank I. De Moortel for helpful discussions. The AIA data used here is courtesy of the SDO (NASA) and AIA consortium. This research has been made possible by the topping-up grant CHARM+top-up COR-SEIS of the BELSPO and the Indian DST. It was partly funded by the IAP P7/08 CHARM and an FWO Vlaanderen Odysseus grant.
Table 3

Periodicity, Exponential Fit Parameters, and the Reduced $\chi^{2}$ Values for all the Data from AIA 193 channel

\begin{tabular}{|c|c|c|c|c|c|}
\hline Structure & $\begin{array}{c}\text { Period } \\
\text { (minutes) }\end{array}$ & $\begin{array}{c}A_{0} \\
\text { (Arbitrary Units) }\end{array}$ & $\begin{array}{c}L_{d} \\
(\operatorname{arcsec})\end{array}$ & $\begin{array}{c}C \\
\text { (Arbitrary Units) }\end{array}$ & $\chi_{\text {red }}^{2}$ \\
\hline Loop 1 & 3.0 & $81.62 \pm 2.39$ & $1.87 \pm 0.10$ & $9.90 \pm 0.43$ & 7.84 \\
\hline Loop 1 & 12.1 & $58.75 \pm 2.72$ & $10.89 \pm 1.53$ & $25.23 \pm 2.60$ & 27.39 \\
\hline Loop 1 & 17.1 & $121.21 \pm 7.62$ & $13.48 \pm 2.61$ & $45.37 \pm 8.72$ & 154.00 \\
\hline Loop 2 & 2.8 & $63.19 \pm 2.82$ & $2.15 \pm 0.17$ & $8.05 \pm 0.54$ & 11.76 \\
\hline Loop 3 & 4.7 & $22.28 \pm 0.47$ & $8.85 \pm 0.45$ & $5.72 \pm 0.26$ & 0.89 \\
\hline Loop 3 & 7.2 & $37.66 \pm 1.31$ & $18.37 \pm 1.86$ & $6.90 \pm 1.57$ & 3.61 \\
\hline Loop 4 & 4.7 & $13.32 \pm 0.65$ & $15.48 \pm 2.23$ & $4.98 \pm 0.77$ & 0.88 \\
\hline Slit 3 & 2.5 & $4.54 \pm 0.04$ & $62.96 \pm 1.72$ & $0.81 \pm 0.03$ & 0.05 \\
\hline Slit 3 & 7.9 & $13.74 \pm 0.20$ & $27.08 \pm 0.68$ & $1.30 \pm 0.05$ & 0.48 \\
\hline Slit 3 & 14.4 & $26.11 \pm 0.65$ & $28.98 \pm 1.26$ & $1.60 \pm 0.17$ & 5.27 \\
\hline Slit 3 & 28.8 & $70.20 \pm 1.39$ & $19.46 \pm 0.62$ & $3.47 \pm 0.26$ & 16.59 \\
\hline Slit 4 & 2.3 & $3.48 \pm 0.05$ & $48.70 \pm 1.59$ & $0.98 \pm 0.03$ & 0.04 \\
\hline Slit 4 & 5.6 & $5.01 \pm 0.10$ & $34.91 \pm 1.38$ & $1.11 \pm 0.04$ & 0.15 \\
\hline Slit 4 & 8.6 & $7.31 \pm 0.13$ & $30.40 \pm 1.03$ & $1.17 \pm 0.04$ & 0.24 \\
\hline Slit 4 & 14.4 & $14.76 \pm 0.31$ & $20.76 \pm 0.72$ & $1.48 \pm 0.07$ & 0.87 \\
\hline Slit 4 & 28.8 & $29.39 \pm 0.67$ & $25.74 \pm 1.02$ & $1.87 \pm 0.17$ & 4.97 \\
\hline Slit 5 & 2.5 & $2.55 \pm 0.03$ & $60.29 \pm 2.05$ & $0.93 \pm 0.02$ & 0.02 \\
\hline Slit 5 & 6.6 & $7.11 \pm 0.13$ & $28.61 \pm 0.92$ & $1.77 \pm 0.03$ & 0.21 \\
\hline Slit 5 & 18.7 & $17.68 \pm 0.26$ & $28.62 \pm 0.74$ & $1.84 \pm 0.07$ & 0.82 \\
\hline Slit 9 & 2.3 & $2.11 \pm 0.03$ & $59.64 \pm 2.32$ & $0.90 \pm 0.02$ & 0.02 \\
\hline Slit 9 & 6.1 & $4.13 \pm 0.10$ & $23.80 \pm 1.00$ & $1.11 \pm 0.02$ & 0.11 \\
\hline Slit 9 & 11.1 & $9.54 \pm 0.23$ & $15.69 \pm 0.59$ & $1.28 \pm 0.04$ & 0.36 \\
\hline Slit 9 & 14.4 & $8.02 \pm 0.26$ & $19.00 \pm 1.01$ & $1.37 \pm 0.05$ & 0.58 \\
\hline Slit 9 & 24.3 & $9.74 \pm 0.19$ & $47.24 \pm 2.14$ & $1.04 \pm 0.10$ & 0.74 \\
\hline Slit 10 & 3.6 & $3.33 \pm 0.07$ & $31.00 \pm 1.44$ & $1.19 \pm 0.03$ & 0.07 \\
\hline Slit 10 & 6.1 & $4.92 \pm 0.12$ & $20.04 \pm 0.82$ & $1.33 \pm 0.03$ & 0.12 \\
\hline Slit 10 & 18.7 & $30.96 \pm 0.87$ & $8.47 \pm 0.37$ & $3.21 \pm 0.12$ & 3.09 \\
\hline
\end{tabular}

\section{APPENDIX}

The exponential fit parameters for all the periods identified in the on-disk and polar data are listed in Tables 2 and 3 for the 171 and 193 channels, respectively. The corresponding reduced $\chi^{2}$ values are also listed as a goodness-of-fit statistic.

\section{REFERENCES}

De Moortel, I. 2009, SSRv, 149, 65

De Moortel, I., \& Hood, A. W. 2003, A\&A, 408, 755

De Moortel, I., \& Hood, A. W. 2004, A\&A, 415, 705

De Moortel, I., Hood, A. W., Gerrard, C. L., \& Brooks, S. J. 2004, A\&A, 425,741

De Moortel, I., Ireland, J., \& Walsh, R. W. 2000, A\&A, 355, L23

De Pontieu, B., \& McIntosh, S. W. 2010, ApJ, 722, 1013

Deforest, C. E., \& Gurman, J. B. 1998, ApJL, 501, L217

Gupta, G. R., Teriaca, L., Marsch, E., Solanki, S. K., \& Banerjee, D. 2012, A\&A, 546, A93

Hood, A. W., Ruderman, M., Pascoe, D. J., et al. 2013, A\&A, 551, A39

Kiddie, G., De Moortel, I., Del Zanna, G., McIntosh, S. W., \& Whittaker, I. 2012, SoPh, 279, 427

King, D. B., Nakariakov, V. M., Deluca, E. E., Golub, L., \& McClements, K. G. 2003, A\&A, 404, L1

Krishna Prasad, S., Banerjee, D., \& Gupta, G. R. 2011, A\&A, 528, L4

Krishna Prasad, S., Banerjee, D., \& Singh, J. 2012a, SoPh, 281, 67

Krishna Prasad, S., Banerjee, D., Van Doorsselaere, T., \& Singh, J. 2012b, A\&A, 546, A50

Lemen, J. R., Title, A. M., Akin, D. J., et al. 2012, SoPh, 275, 17

Marsh, M. S., De Moortel, I., \& Walsh, R. W. 2011, ApJ, 734, 81

Marsh, M. S., Walsh, R. W., \& Plunkett, S. 2009, ApJ, 697, 1674

Nakariakov, V. M., Verwichte, E., Berghmans, D., \& Robbrecht, E. 2000, A\&A, 362,1151

Nishizuka, N., \& Hara, H. 2011, ApJL, 737, L43

Ofman, L., Nakariakov, V. M., \& Sehgal, N. 2000, ApJ, 533, 1071

Ofman, L., \& Wang, T. 2002, ApJL, 580, L85

Ofman, L., Wang, T. J., \& Davila, J. M. 2012, ApJ, 754, 111

Pascoe, D. J., Hood, A. W., de Moortel, I., \& Wright, A. N. 2012, A\&A, 539, A37

Pesnell, W. D., Thompson, B. J., \& Chamberlin, P. C. 2012, SoPh, 275, 3

Tian, H., McIntosh, S. W., \& De Pontieu, B. 2011a, ApJL, 727, L37 
Tian, H., McIntosh, S. W., De Pontieu, B., et al. 2011b, ApJ, 738, 18

Tian, H., McIntosh, S. W., Wang, T., et al. 2012, ApJ, 759, 144

Tsiklauri, D., \& Nakariakov, V. M. 2001, A\&A, 379, 1106

Uritsky, V. M., Davila, J. M., Viall, N. M., \& Ofman, L. 2013, ApJ, 778, 26

Van Doorsselaere, T., Wardle, N., Del Zanna, G., et al. 2011, ApJL, 727, L32
Wang, T., Ofman, L., \& Davila, J. M. 2013, ApJL, 775, L23

Wang, T. J., Ofman, L., Davila, J. M., \& Mariska, J. T. 2009, A\&A, 503, L25

Wang, T. J., Solanki, S. K., Curdt, W., Innes, D. E., \& Dammasch, I. E. 2002, in ESA Special Publication, Vol. 508, From Solar Min to Max: Half a Solar Cycle with SOHO, ed. A. Wilson (Noordwijk: ESA), 465 